La calidad de las editoriales universitarias en los institutos técnicos y tecnológicos de educación superior de la ciudad de Quito

Número Publicado el 05 de julio de 2018

DOI: $10.23857 /$ dc.v4i3.794

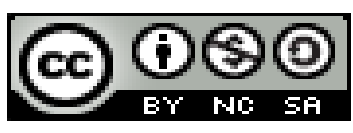

Ciencias sociales y políticas

Artículo original

\title{
La calidad de las editoriales universitarias en los Institutos Técnicos y Tecnológicos de Educación Superior de la ciudad de Quito
}
The quality of the university publishers in the Technical and Technological Institutes of Higher Education of the city of Quito

\section{A qualidade das editoras universitárias nos Institutos Técnicos e Tecnológicos de Ensino Superior da cidade de Quito}

Vanessa P. Quishpe-Morocho I

vanessaquishpe@hotmail.com

Miguel García-Jiménez II

mgjmolina@hotmail.com

Recibido: 14 de enero de 2018 * Corregido: 25 de febrero de 2018 * Aceptado: 07 de junio de 2018

I. Ingeniera en Ciencias de la Información y Bibliotecología, Universidad Tecnológica Israel, Quito, Ecuador.

II. PhD en Derecho Público, Universidad Tecnológica Israel, Quito, Ecuador. 
La calidad de las editoriales universitarias en los institutos técnicos y tecnológicos de educación superior de la ciudad de Quito

\title{
Resumen
}

Las revistas científicas constituyen los principales canales de comunicación, difusión y certificación de los resultados de las investigaciones de la comunidad científica tanto en Ecuador como sus pares en el mundo. Escribir artículos de investigación es una de las actividades actuales del docente investigador, de todas las Instituciones de Educación Superior e Institutos Técnicos y Tecnológicos de Quito. En este sentido, investigadores se proponen un objetivo, que es divulgar los resultados, parciales o finales, de sus investigaciones, tanto en revistas arbitradas e indexadas, nacionales como internacionales. En Ecuador ha crecido notablemente la edición de revistas científicas, sin embargo, se plantea el problema de la calidad de estos órganos de difusión, por la ausencia de una regulación específica. Esta investigación se enfocará en la realización de un diagnóstico sobre el estado de las políticas editoriales, y se propone el método descriptivo que permitirá ordenar los resultados de las observaciones de la realidad obteniendo como resultado la identificación de las fallas en las políticas y obtener como efecto la propuesta de mejora en las mismas.

Palabras clave: políticas; editoriales; tecnológicos; investigación; artículos.

\begin{abstract}
Scientific journals are the main channels of communication, dissemination and certification of research results of the scientific community both in Ecuador and their peers in the world. Writing research articles is one of the current activities of the research teacher, of all the Institutions of Higher Education and Technical and Technological Institutes of Quito. In this sense, researchers propose an objective, which is to disclose the results, partial or final, of their research, both in refereed and indexed journals, national and international. In Ecuador, the edition of scientific journals has grown considerably, however the problem of the quality of these organs of diffusion is raised, due to the absence of a specific regulation. This investigation will focus on the realization of a diagnosis on the state of the editorial policies, and the descriptive method is proposed that will allow to order the results of the observations of the reality obtaining as a result the identification of the failures in the policies and obtain as effect the proposal for improvement in them.
\end{abstract}

Keywords: policies; editorials; technological; research; articles. 
La calidad de las editoriales universitarias en los institutos técnicos y tecnológicos de educación superior de la ciudad de Quito

\section{Resumo}

As revistas científicas são os principais canais de comunicação, divulgação e certificação dos resultados de pesquisa da comunidade científica, tanto no Equador quanto em seus pares no mundo. Escrever artigos de pesquisa é uma das atividades atuais do professor pesquisador, de todas as Instituições de Ensino Superior e Institutos Técnicos e Tecnológicos de Quito. Nesse sentido, os pesquisadores propõem um objetivo, que é divulgar os resultados, parciais ou finais, de suas pesquisas, tanto em revistas indexadas quanto indexadas, nacionais e internacionais. No Equador, a edição de revistas científicas cresceu consideravelmente, mas o problema da qualidade desses órgãos de difusão é elevado, devido à ausência de uma regulamentação específica. Esta investigação incidirá sobre a realização de um diagnóstico sobre o estado das políticas editoriais, sendo proposto o método descritivo que permitirá ordenar os resultados das observações da realidade obtendo como resultado a identificação das falhas nas políticas e obtendo como efeito a proposta de melhoria neles.

Palavras chave: políticas; editoriais; tecnológico; pesquisa; artigos.

\section{Introducción}

La investigación como simple proceso de comprobación o refutación de los supuestos, teorías fenómenos, es importante, ya que permite situar a un área del conocimiento a la vanguardia demostrando lo que tiene utilidad y lo que no, según Vélez (2001), afirma que la investigación es el término es el "proceso sistemáticamente ordenado, cuyo objetivo es la demostración de hipótesis o la confirmación y desarrollo de teorías".

Estos hallazgos por sí solos no tienen relevancia si no se comunican, por lo tanto, la publicación de artículos es una actividad fundamental principalmente en las universidades y centros de investigación de Gil \& Sardinha (2011).

Ecuador desde el 2010 emitió la Ley Orgánica de Educación Superior-LOES conjuntamente con el Reglamento de Régimen Académico y el Reglamento de Institutos y Conservatorios Superiores, directrices que han permitido estructurar el sistema de educación superior consolidado, logrando hacer que se tome un rumbo distinto en la organización y reestructuración administrativa-docente de las universidades e institutos tecnológicos del país, a pesar de existir normativa superior lamentablemente se ha evidenciado inconsistencias o aclaratorias en lo que respeta a la creación de revistas científicas de los institutos superiores, técnicos, tecnológicos y pedagógicos en específico. 
La calidad de las editoriales universitarias en los institutos técnicos y tecnológicos de educación

Situación que ha traído como consecuencia que los 72 Institutos superiores, técnicos, tecnológicos y pedagógicos de la ciudad de Quito trabajen de forma desorganizada e incluso le han dado poca importancia a la publicación de artículos y sobre todo a la creación de revistas científicas de calidad, que permitan conocer las investigaciones y productos obtenidos de sus proyectos científicos o de la vinculación con la sociedad que han realizado los estudiantes.

Es por esta causa, que este se ha convertido en el nodo de esta investigación, la forma en que las editoriales asumen, desde la perspectiva de la calidad, la publicación de los artículos científicos, en los tecnológicos de educación superior porque tal como dice Guédon (2011) la función de las revistas científicas es convertirse en una especie de registro social de invenciones e innovaciones.

En las revisiones estadísticas de la producción de artículos científicos en Ecuador casi no se visibiliza los resultados de esta producción científicas de los Institutos Técnicos y Tecnológicos de Quito; y por eso, es que no ha sido posible cuantificar estas investigaciones que han devenido en artículos científicos; y como plantea, Piccone y Jousset (2011) hablando de la visibilización de las instituciones de educación superior, indican que los rankings mundiales de universidades (en este caso institutos y Tecnológicos) surgen en gran medida de los intercambios, trama de citas y número de publicaciones que se establecen en cada ámbito del conocimiento.

Hay factores que inciden en esta invisibilización del trabajo investigativo en estos centros de Educación Superior, por un lado, se evidencian falencias investigativas y por el otro es evidente la necesidad de normalizar las creaciones de revistas, específicamente en la calidad de los artículos, en las revisiones bibliográficas, entre otros productos obtenidos de investigaciones.

Algunos datos estadísticos nos permiten evaluar, a través de los años, la producción de las publicaciones científicas en Ecuador: 
La calidad de las editoriales universitarias en los institutos técnicos y tecnológicos de educación superior de la ciudad de Quito

Tabla 2. Producción científica y tasa de crecimiento comparada 2000-2013

Pc: Producción citable (incluye artículos, resúmenes y comunicaciones a congresos); Ci: Citas; Tc Tc: Tasa de crecimiento. Datos de SJR (2014)

\begin{tabular}{|c|c|c|c|}
\hline & \multicolumn{2}{|c|}{ Ecuador } & \multirow[b]{2}{*}{ TcEcu } \\
\hline & Pc & $\mathrm{Ci}$ & \\
\hline 2000 & 123 & 3.044 & \\
\hline 2001 & 112 & 3.103 & $-0,09$ \\
\hline 2002 & 144 & 2.927 & 0,29 \\
\hline 2003 & 191 & 3.592 & 0,33 \\
\hline 2004 & 201 & 5.157 & 0,05 \\
\hline 2005 & 247 & 4.825 & 0,23 \\
\hline 2006 & 287 & 5.427 & 0,16 \\
\hline 2007 & 326 & 4.318 & 0,14 \\
\hline 2008 & 385 & 6.979 & 0,18 \\
\hline 2009 & 463 & 5.076 & 0,20 \\
\hline 2010 & 424 & 4.457 & 0,08 \\
\hline 2011 & 444 & 2.579 & 0,05 \\
\hline 2012 & 578 & 1.880 & $\mathbf{0 , 3 0}$ \\
\hline 2013 & 638 & 406 & $\mathbf{0 , 1 0}$ \\
\hline Total & 4.563 & 53.770 & \\
\hline
\end{tabular}

Fuente: Álvarez, y Pérez (2015).

Si bien es cierto que en esta investigación se observa un incremento importante tanto en la producción investigativa como en su publicación también es preocupante que al desglosar estas estadísticas los Institutos Técnicos y Tecnológicos de Quito casi nunca son tomados en cuenta.

Esto se puede evidenciar por las áreas donde se centra la mayor producción de artículos científicos, según Álvarez y Pérez (2015) las áreas que más tienen editoriales especializadas agricultura el 21,79\% , ingeniería con un índice similar al de la agricultura, la medicina con el 20,6\%., la producción de bioquímica, genética y biología molecular se sitúa en un 8,52\%, seguido por ciencias medioambientales con un $8,24 \%$ y ciencias sociales con un 5,68\%; áreas que en su mayoría no son áreas de conocimientos impartidas en los Institutos Técnicos y Tecnológicos 
La calidad de las editoriales universitarias en los institutos técnicos y tecnológicos de educación superior de la ciudad de Quito

Esto reafirma la necesidad de aumentar y mejorar la cantidad y la calidad de las publicaciones, tanto en el contenido como en la forma. Y es allí donde es importante definir lo que es una editorial

Se califica como editoriales universitarias «a aquellas editoriales que pertenecen a las instituciones de educación superior, que cumplen funciones de edición e impresión y que destinan sus productos culturales para uso académico de las mismas y, también, para sectores extra-universitarios» (Rama, Uribe y De Sagastizábal, 2006).

Sobre el primer aspecto, se deben tener estrategias muy claras para velar por su calidad: el arbitraje por parte de expertos y la revisión ortográfica y de estilo por parte de un corrector.

Existe, además, la evaluación de la comunidad académica o científica, según Kuhn (1962) es un grupo de científicos que comparten un paradigma que, aunque está fuera del dominio de la editorial universitaria, ejerce tal vez el control de calidad más importante en su condición de lector-consumidor.

Es así, como estudiar ciertos aspectos relacionados con la forma y fondo de las revistas resulta muy necesario, debido a que estas investigaciones se transforman en especies de monitoreo de las prácticas que están llevando a cabo estos dispositivos difusores del nuevo conocimiento que generan los Institutos Técnicos y Tecnológicos de Quito, los respectivos programas, centros o institutos de investigación.

Es innegable que mientras mejor sea el conocimiento que se publica, permite mejor y mayor visibilidad, facilita el de acceso y la credibilidad nacional e internacional de la publicación científica, todo esto incide en la mejora de la docencia que se entrega a los ciudadanos y mejores probabilidades de progreso y desarrollo tendrán los respectivos países.

\section{Metodología}

Para realizar la investigación se aplicarán los procedimientos lógicos de la deducción (para desprender de enunciados generales otros particulares), inductivos (para generalizar observaciones particulares), el análisis (descomposición de un todo en sus partes), la síntesis (posterior al análisis, relacionar las partes para observar cómo se relacionan en el todo recompuesto en el pensamiento), la comparación (de acuerdo a criterios explícitos y homogéneos). 
La calidad de las editoriales universitarias en los institutos técnicos y tecnológicos de educación

Estas operaciones lógicas son complementarias entre ellas y son propias de todos los procesos racionales de producción de conocimientos. Servirán para describir, construir explicaciones, procesar los datos del trabajo de campo de manera cuantitativa y cualitativa.

La investigación descriptiva que permitirá ordenar los resultados de las observaciones de la realidad obteniendo como resultado la identificación de las fallas en las políticas y obtener como efecto la propuesta de mejora en las mismas.

La investigación cualitativa permitirá trabajar con los registros narrativos de los fenómenos que son estudiados mediante técnicas como la observación participante. Para ilustrar este análisis hay que construir un modelo cualitativo de su gestación y tendencias, identificando de manera precisa (empleando indicadores cuantitativos, cuando ello sea necesario) cuáles causas son flujos, acumulaciones y reglas.

Para el trabajo de campo, se aplicará el método Delphi, clasificado generalmente como un método cualitativo. Este método sirve para establecer un consenso entre las opiniones y las intuiciones de un grupo de expertos, mediante la estructuración de un proceso de comunicación para tratar un tema específico de cierta complejidad. Se instrumenta a través de sucesivas rondas de entrevistas, con las cuales se les pregunta acerca de los elementos de una situación determinada, perspectivas de solución, su futuro y posibles soluciones; en este caso la baja producción de publicaciones científicas en los institutos superiores técnicos y tecnológicos de Quito, Ecuador, en un mundo donde este tipo de medio indica un desarrollo específico de la actividad de la comunidad científica del país.

Se harían dos rondas de preguntas, con el fin de identificar los puntos donde pueda producirse una convergencia de opiniones e inferir eventuales consensos. En la primera ronda, se aplicará una entrevista a los expertos seleccionados, para conocer sus opiniones, intuiciones y apreciaciones acerca de a) identificación de "nudos problemáticos", obstáculos, necesidades, requerimiento, para lograr mejorar la producción y visibilidad de revistas científicas, b) sugerencias de objetivos, visiones y misiones, medidas, decisiones, reglas o normas, que puedan tomarse o elaborarse para desarrollar una adecuada política editorial,) 
La calidad de las editoriales universitarias en los institutos técnicos y tecnológicos de educación

ideas para resolver problemas específicos de organización institucional, designaciones, que se puedan resolver y aplicar a nivel nacional, regional y en cada instituto.

En la segunda ronda del método Delphi, se informará a los expertos participantes acerca de las opiniones, consideraciones y sugerencias que han tenido mayor convergencia de opiniones, se les interrogará acerca de su apreciación respecto a estos puntos de convergencia y, en caso de divergencia, se les solicitará explicaciones acerca de sus posiciones opuestas o diversas.

Este trabajo pretende ser una herramienta de información para el análisis de la producción científica de los Institutos Técnicos y Tecnológicos de Quito, cuyos resultados puedan contrastarse con los estudios de otros autores, y a su vez, un instrumento de ayuda adicional en la toma de decisiones de autoridades universitarias, investigadores y Editoriales Universitarias

El método de Planificación Estratégica Situacional (PES) distingue, en este juego complejo de elementos que es la realidad, entre acumulaciones (que son capacidades, aptitudes y competencias adquiridas de acuerdo a cierta trayectoria de acción y, en definitiva una historia específica), flujos (acciones, operaciones, "jugadas") y reglas (regularidades o normalidades, establecidas o no, bien definidas o difusas, ocasionadas en parte por estructuras o relaciones estables que se han reforzado por causas determinables). Si seguimos con la metáfora del juego, las causas inmediatas del marcador del juego son las jugadas, que llamamos flujos. Para producir jugadas se requieren capacidades de producción, que las identificamos como acumulaciones. Estas acumulaciones son capacidades de producción de flujos o jugadas que poseen o utilizan los jugadores. Pero las jugadas y las acumulaciones pertinentes y válidas son las que permiten las reglas del juego. Esas acumulaciones, flujos y reglas pueden estar o no bajo nuestro control como planificadores u operadores en la realidad o, incluso, pueden provenir de otros “juegos" o realidades (Huertas, 2009).

\section{Resultados}

Es necesario sustentar la necesidad de creación de una política pública editorial que permita medir la calidad de lo que produce estas editoriales para los Institutos superiores, técnicos, tecnológicos y pedagógicos, tomando en cuenta que según Adabal y Rius (2006) los científicos publican para contribuir al progreso social de la ciencia. 
La calidad de las editoriales universitarias en los institutos técnicos y tecnológicos de educación

Con cifras aportadas por la base de datos SCOPUS Se ha realizado un diagrama de medición sobre el impacto de la política de investigación científica, se ha tomado que establece parámetros de calidad internacionalmente aceptados. En la mencionada base de datos, el Ecuador entre los años 2012 y 2015, presenta un importante incremento en su producción científica, pasando de 639 publicaciones indexadas en 2012 a 1.237 en 2015 (Scopus, 2017).

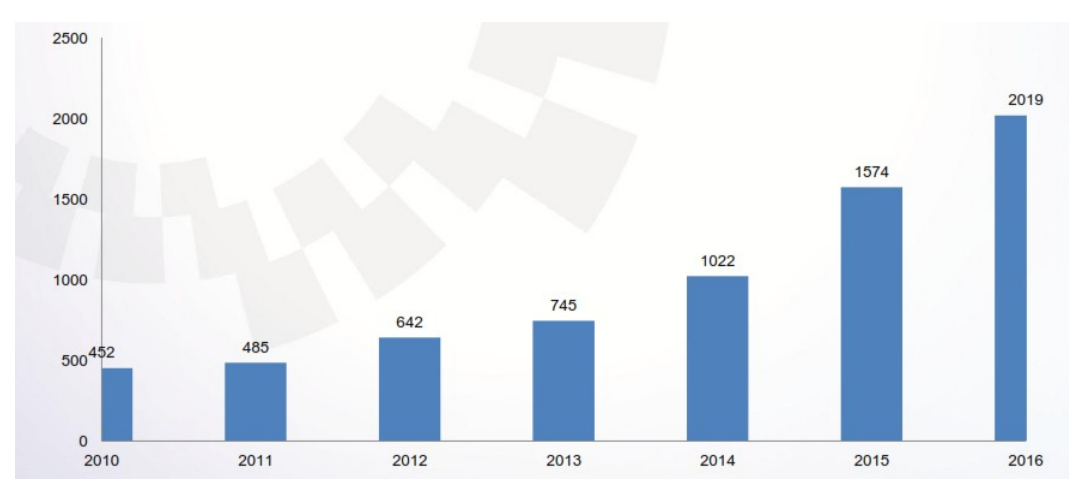

Gráfico 1. Total, de publicaciones indexadas en Scopus Fuente: SCOPUS (2017).

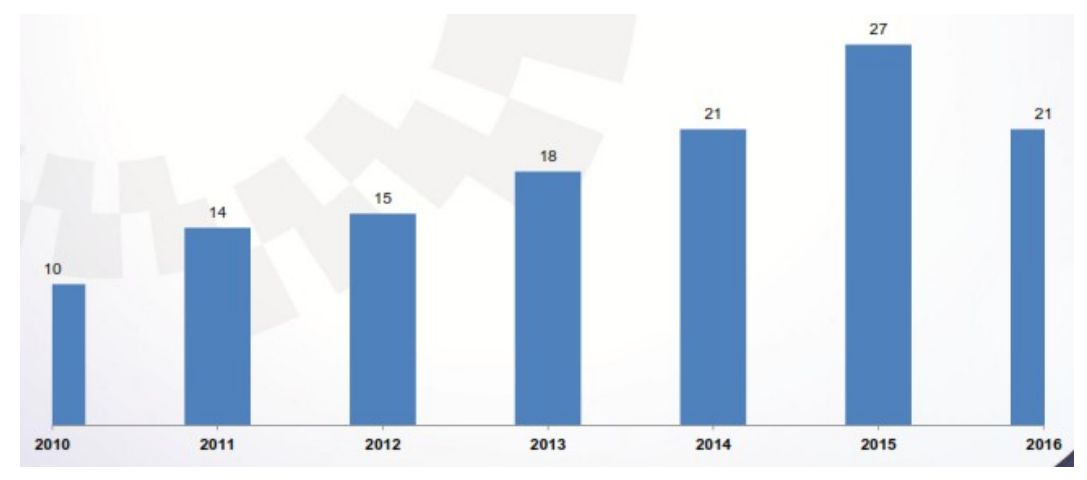

Gráfico 2. Número de Instituciones de Educación Superior que publican en Scopus Fuente: SCOPUS (2017).

Aunque en estas estadísticas no hay un desglosamiento por instituciones de Educación Superior que publican artículos científicos en Ecuador, la situación de los Institutos Tecnológicos se evidencia cuando el Consejo de Educación Superior emite un decreto para elevar la categoría del nivel de instrucción de estas instituciones, en el año 2016, logrando que los estudiantes obtengan posterior a la finalización de sus estudios en cualquier instituto técnico, el título del tercer nivel. 
La calidad de las editoriales universitarias en los institutos técnicos y tecnológicos de educación

Al obtener el rango de Educación superior los tecnológicos se ven obligados a cumplir con uno de los postulados que certifiquen y validen su cambio de categoría y es el postulado de la investigación. En vista a esta resolución emitida por el CES se debe dar importancia a la creación de revistas científicas que contengan los productos investigativos de los estudiantes, factor que influye en el incremento de apariciones de revistas indexadas (Álvarez \& Junsoca, 2014).

\section{Discusión}

La evaluación de la actividad científica y de la productividad de los investigadores es una cuestión de interés desde muchas perspectivas distintas: la financiación de la investigación, la promoción de los investigadores, la recompensa de la actividad de investigación, la formulación de políticas de investigación y la toma de decisiones relacionadas con tales políticas, la planificación estratégica de la actividad universitaria, la negociación salarial en los casos en que ésta se hace directamente entre el investigador y la organización contratante, la dotación de plazas de profesorado universitario y la promoción del profesorado y la concesión de becas, entre otras (García-Pérez, 2000).

Toda esta política de mejorar las condiciones de la Educación Superior ha contribuido a un ascenso social donde se pretende que la participación ciudadana tenga acceso y llegue a formar parte en la construcción del ese conocimiento.

A ese reto es que los Institutos Técnicos y Tecnológicos de Quito y la investigación académica, debe responder por medio de acertados programas para la formación de investigadores; y luego, cristalizar proyectos editoriales que le permitan a estas instituciones avanzar en la transformación social, gracias a los conocimientos adquiridos e intercambiados por medio de ese vaso comunicante que representan las revistas científicas.

«La editorial universitaria debe ser una empresa, aunque haya nacido del medio universitario y debe cumplir exigencias de racionalidad económica propias de toda compañía si no quiere convertirse en un lastre que tarde o temprano, no podrá ser soportado por la universidad» (Rama, Uribe y De Sagastizábal, 2006)»

Pero también, es una prioridad que las Institutos Técnicos y Tecnológicos de Quito sepan orientar esas inversiones en formación de recursos humanos de primera línea a nivel nacional e internacional, y en la consolidación de infraestructura de producción editorial que le permita rentabilizar el capital 
La calidad de las editoriales universitarias en los institutos técnicos y tecnológicos de educación superior de la ciudad de Quito

intelectual de los investigadores a través de convenios internacionales de intercambios científicos; optimizar los mecanismos de intercambios en físico y electrónicos de las revistas, así como en la creación de nuevos grupos o comunidades de investigadores (as) y de otras revistas científicas.

Si las políticas de investigación se acoplan a las políticas editoriales, de acuerdo a objetivos comunes o complementarios, la fortaleza editorial de los científicos para procesar la producción del conocimiento científico será cada vez más necesaria e imprescindible. Y será, por esa misma esencia que los Institutos Técnicos y Tecnológicos de Quito, de cumplirse esta meta, lleguen a convertirse en libros de esos saberes, es decir, en la biblioteca, enciclopedia, ciencia de la racionalidad, ética, moral, humanísticas, de conocimientos. Y es lo que por tradición histórica refleja la cultura o civilización que porta toda institución de Educación Superior, como dijo Alfredo Bryce Echenique (2002) "en los albores del siglo XXI, el libro sigue con más vigencia que nunca, no en vano deja tras de sí muchos años y generaciones como contenedor y transmisor de cultura".

Las publicaciones científicas, es decir, libros y revista arbitradas, son la consecuencia natural de la esencia de los centros de Educación superior, Institutos Técnicos y Tecnológicos, estas van destinadas a la comunicación entre la comunidad de investigadores, sin la cual ni la comunidad se ensancha ni los investigadores validan sus conocimientos.

Una de las fortalezas de una Universidades o Tecnológicos de primer orden (aun en el tercer mundo), es disponer de un sello editorial que le permita acceder al mercado del libro para un público especializado, pero que no cierre sus fronteras al público general. El saber es un espacio del dominio público de cualquier lector, y el principio esencial de la universalidad del conocimiento es compartir libremente el conocimiento con otro. Para Sierra (1991) las editoriales universitarias están inmersas en una institución cuyo desempeño social tiene funciones específicas relacionadas con el saber: la docencia, la investigación y la difusión cultural.

Para poder cubrir la demanda que presiona sobre la difusión de los conocimientos, un proyecto editorial debe poseer toda una política de publicaciones que responda a las expectativas de investigadores que desean ver en red la circulación de los resultados de sus investigaciones.

No se trata solamente de imprimir en papel y tinta unas cuantas hojas en formato de artículos o libros. El desafío es mucho mayor en una sociedad de conocimientos globalizados, donde es imprescindible 
La calidad de las editoriales universitarias en los institutos técnicos y tecnológicos de educación superior de la ciudad de Quito

disponer de altas tecnologías de información y comunicación para estar al día con los resultados de las ciencias.

Las editoriales, bibliotecas, virtuales son en este momento una objetiva realidad, que no se puede ignorar: eso sería sucumbir al atraso y permanecer en la decadencia. La investigación en red o en línea, requieren de publicaciones de igual manera para un mundo cibernético que requiere ser compartido, para estar en tiempo virtual en cualquier espacio de la realidad humana. Retomando la sentencia de Fernando García Garanz (1998) “el ordenador conectado a la red será, es, un instrumento de trabajo para dar acceso a la biblioteca más grande del mundo".

\section{Conclusión}

Dado el auge de revista y por consiguiente de editoriales, es conveniente analizar a profundidad las normativas vigentes por parte del sistema del Estado que administra la educación, con el propósito de proponer políticas coherentes que permitan evaluar y normar el enfoque del conocimiento editorial, garantizar la normalización editorial entre la comunidad de los institutos del país y los entes reguladores y las Editoriales Universitarias.

En este marco, surge la necesidad de contribuir a la construcción de normativas para mejorar la calidad de la política editorial de las revistas de divulgación académica en los Institutos Técnicos y tecnológicos de la ciudad de Quito.

Con el fin de cumplir este propósito se propone elabora un plan o propuesta sistematizada que lleve las siguientes pautas:

Visión y misión de la política editorial científica. Definiciones básicas.

Estructura organizativa directiva a nivel nacional (Consejo Nacional editorial) así como estructuras análogas a nivel de cada instituto, que se encargarían de gerenciar, planificar, ejecutar, hacer seguimiento y evaluar la actividad.

Líneas de productos editoriales: libros de texto de asignaturas, revistas científicas, libros originales científicos, tesis de grado, monografías, memorias de congresos científicos, colecciones de textos traducidos, antologías. 
La calidad de las editoriales universitarias en los institutos técnicos y tecnológicos de educación superior de la ciudad de Quito

Criterios académicos y editoriales para aprobar publicaciones. Reglamentaciones de cada revista científica creada. Protocolos para la recepción de originales, evaluación, selección y publicación.

Plan anual de publicaciones.

Actividades de formación de autores. Disposiciones de estímulo a los profesores y estudiantes para publicar (ventajas para ascenso, estímulos). Derechos de autor.

Criterios para la promoción, distribución y comercialización de los productos.

Creación de índice nacional de revistas científicas y biblioteca virtuales.

Para las editoriales que hacen vida en los tecnológicos es menester asumir la visión de uno de los índices más importante que existe hoy día en el mundo académico virtual, el Proyecto SCIELO, proyecto que se propone promover el perfeccionamiento de la comunicación científica nacional en todos sus aspectos, buscando identificar, estimular y desarrollar una colección-núcleo de revistas científicas, cuyo estándar de calidad alcance al de las revistas científicas internacionales de primera línea.

\section{Referencias Bibliográficas}

Adabal, E., \& Rius, L. (2006). Revistas Científicas Digitales: Características e Indicadores. Revista de Universidad y Sociedad del Conocimiento. 3 (1). [En línea] Disponible en: http://www.uoc.edu/rusc/3/1/dt/esp/abadal_rius.pdf

Álvarez, L., \& Juncosa, J. (2014). La evaluación y acreditación de la educación superior ecuatoriana y la producción editorial. Universitas, Revista de Ciencias Sociales y Humanas, 21, Pp. 163-181 [en línea], Disponible en: http://www.redalyc.org/pdf/4761/476147261008.pdf

Álvarez, P y Pérez, M. (2015). Análisis de la producción y de la visibilidad científica de Ecuador en el contexto andino (2000-2013). El profesional de la Información, 24 (5) [en línea], Disponible en: https://recyt.fecyt.es/index.php/EPI/article/view/epi.2015.sep.07

Bryce, A. (2002). El placer de la palabra escrita. El Dominical de El Comercio de Lima. Lima, Perú. García, F. (1998). Libros en Internet. Madrid: Editorial Espasa Calpe S.A.

Garcia-Pérez, M. (2000). Assessors' odd listings don’t inspire confidence. Nature, 406 (6794), Pp. 361-364. 
La calidad de las editoriales universitarias en los institutos técnicos y tecnológicos de educacion superior de la ciudad de Quito

Gil, C., \& Sardinha, A. (2011). H-Index of the citing articles: A contributionto the evaluation of scientific production of experienced researched. Revista Brasileira de Medicina do Esporte, 17 (5), Pp.358-362.

Guédon, J.-C. (2011). Beyond core journal and licenses: the paths to reform scientific publishing. [en línea], Disponible en: http://www.arl.org/newsltr/218/guedon.html

Huertas, F. (2009). El método PES. Planificación Estratégica Situacional. Entrevista con Carlos Matus. Santiago. Chile: Editorial CERES. Fundación Altadir.

Kuhn, T. (1962). La Estructura de las Revoluciones Científicas. México: Fondo de Cultura Económica.

Piccone, M., \& Jousset, M. (2011). Impacto y visibilidad de las revistas científicas. Buenos Aires: Biblioteca Nacional.

Rama, C., Uribe, R., \& De Sagastizábal, L. (2006). Las Editoriales Universitarias en América Latina. [en línea], Disponible en: http://cerlalc.org/wpcontent/uploads/publicaciones/olb/PUBLICACIONES_OLB_\%20Las-editoriales-universitarias-enAmerica-Latina_v1_010106.pdf

Scopus. (2017). Data de publicaciones. [en línea], Disponible en: http://www.scopus.com Sierra, J. (1991). Manual de gestión y mercadeo para editoriales universitarias. México: Centro Regional para el Fomento del Libro en América Latina y el Caribe.

Vélez, S. (2001). Apuntes de metodología de la investigación. Medellín: Departamento de Ciencias Básicas Universidad EAFIT. 